\title{
Midisuperspace Supersymmetric Quantum Cosmology
}

\author{
Alfredo Macías* and Abel Camachd ${ }^{+}$ \\ Departamento de Física, Universidad Autónoma Metropolitana-Iztapalapa, \\ A.P. 55-534, México D.F. 09340, México \\ Jutta Kunz \\ Department of Physics, \\ Carl von Ossietzky University Oldenburg, \\ D-26111 Oldenburg, Germany \\ Claus Lämmerzah $\$$ \\ ZARM, University of Bremen, Am Fallturm, 28359 Bremen, Germany
}

(Dated: November 28, 2018)

\begin{abstract}
We investigate the canonical quantization in the framework of $N=1$ simple supergravity for the case of a very simple gravitational midisuperspace described by Gowdy $T^{3}$ cosmological models. We consider supersymmetric quantum cosmology in the mentioned midisuperspace, where a matrix representation for the gravitino covector-spinor is used. The full Lorentz constraint and its implications for the wave function of the universe are analyzed in detail. We found that there are indeed physical states in the midisuperspace sector of the theory in contrast to the case of minisuperspace where there exist no physical states.
\end{abstract}

File: gowsg5.tex; 30.11.2007

PACS numbers: 04.60.Kz, 04.65.+e, 12.60.Jv, 98.80.Hw

\footnotetext{
*Electronic address: amac@xanum.uam.mx

${ }^{\dagger}$ Electronic address: acq@xanum.uam.mx

${ }^{\ddagger}$ Electronic address: kunz@theorie.physik.uni-oldenburg.de

§Electronic address: laemmerzahl@zarm.uni-bremen.de
} 


\section{INTRODUCTION}

According to Misner [1, 2], quantum cosmology is the evolution of cosmological spacetimes as trajectories in the finite dimensional sector of superspace, the so called minisuperspace, related to the finite number of parameters that describe $t=$ const. slices of the models and the quantum version of such models, respectively. Taking the metric of a cosmological model which is truncated by an enormous degree of imposed symmetry, and plugging it into a quantization procedure cannot give an answer that could be consider in any way as a quantum gravity solution. What is being done in quantum cosmology, is the assumption that one can represent a metric as a series expansion in space-dependent modes, where the cosmological minisuperspace model is the homogeneous mode, and the cosmological midisuperspace model is the first non-homogeneous mode. This artificial "freezing" of the modes before quantization is an obvious violation of the uncertainty principle and cannot lead to an exact solution. However, the results of applying this untenable quantization procedure have always seemed to predict a rather reasonable and internally consistent behavior of the universe that it has been difficult to believe that it does not have any physical meaning.

After the invention of supergravity by Freedman, Nieuwenhuizen, and Ferrara [3] , Teitelboim [4, 5, 6] showed that this theory provides a natural classical square root of gravity á la Dirac. Taking the square root of the constraints amounts to take the square root of the corresponding quantum equations, introducing spin in a natural way. Thereby, the total number of constraints of the theory increases. Besides the constraints of the original theory, there appear now new constraints (the square roots) closing under anticommutation. The complete set of constraints forms a graded algebra [5, 6]. The role of the Dirac squareroot will be played by the new constraints. Furthermore, the local supersymmetry of the action should have profound consequences upon the resulting quantum theory, for example, the supersymmetric constraints will provide a Dirac square root of the second-order Wheeler-DeWitt equation governing the dynamics of the wave function of the universe.

The classical field equations arising from the $N=1$ supergravity Lagrangian were derived by Pilati [7] by using the canonical formalism. There are constraints for each of the gauge symmetries contained in the theory, i.e., spacetime, diffeomorphisms, local Lorentz invariance, and supersymmetry. One important result that follows from the analysis of the field equations is that the Cartan relation relates the torsion tensor and the Rarita-Schwinger 
gravitino field, so that it can be used to eliminate the torsion tensor from the theory.

The canonical quantization of supergravity is performed in general by applying Dirac's procedure for constrained systems. According to it, quantization is performed by choosing a foliation for spacetime, i.e., a $(3+1)$ decomposition of the canonical theory, in which the Lagrange multipliers are the normal components constraining the symmetry generators of the corresponding gauge fields. Of course, all the constraints should annihilate the ground state of the wave function. For the supergravity case, there are three different constraints in the problem, namely, the generators $\mathcal{H}_{\mu}$ of the translations (Hamiltonian and diffeomorphism), the generators $\mathcal{J}_{\mu \nu}$ of local Lorentz rotations and the Majorana spinor (Fermionic functions) supersymmetric generators $\mathcal{S}$. The Lagrange multipliers constraining these generators are the normal components $e^{A}{ }_{0}, \omega_{0 A B}$, and $\bar{\Psi}_{0}$, of the coframe, connection and gravitino field, respectively.

It turns out [5] that the Hamiltonian constraint is identically satisfied once the supersymmetric constraint is fulfilled since they satisfy the relation $\left\{\mathcal{S}(x), \overline{\mathcal{S}}\left(x^{\prime}\right)\right\}=\gamma^{A} \mathcal{H}_{A} \delta\left(x, x^{\prime}\right)$. Accordingly, only the Lorentz and supersymmetric constraints are the central issue of the quantization problem.

The gravitino field appearing in the constraints can be realized in two different ways, namely, by differential operators or by matrices as in the Dirac equation. In this work we will use a matrix representation, á la Dirac, for the gravitino field and since its corresponding momenta are proportional to Rarita-Schwinger field itself, we will not rename them as it happens in the differential operators approach [8].

It is important to stress that general relativity, and therefore supergravity, does not seem to possess a natural time variable, while quantum theory relies quite heavily on a preferred time [9]. Since the nature of time in quantum gravity is not yet clear, the classical constraints of canonical supergravity do not contain any time parameter, after applying to them the canonical quantization procedure. Therefore, it is needed a kind of internal time, which is fixed by means of a gauge choice, or by a classical solution to drive the dynamical behavior of the resulting quantum theory [10].

As mentioned above, the minisuperspace is often known as the homogeneous cosmology sector, infinitely many degrees of freedom are artificially frozen by symmetries. This reduction is so drastic that only a non-physical finite number of degrees of freedom is left. The requirement of homogeneity restricts the allowed hypersurfaces to the leaves of a privileged 
foliation, which is labelled by a single internal time variable, it is usually the volume. One can parameterize such hypersurfaces of homogeneity by the standard Euler angles coordinates and characterize the spatial metric uniquely by three real parameters.

The supersymmetric approach to quantum cosmology was first introduced by Macías et al. [8], and means the study of $N=1$ quantum supergravity models restricted to the homogeneous minisuperspace sector of the Wheeler's superspace as direct generalizations of standard Wheeler-De-Witt quantum cosmological models. The standard approach to quantum cosmology consists in a canonical quantization of homogeneous minisuperspace model, which is obtained by imposing certain symmetry conditions on the metrics allowed on the spacelike slices of the universe [11, 12]. The dynamics of the system is governed by the Wheeler-DeWitt equation which is a second order Klein-Gordon-like differential constraint equation for the state function of the universe [13]. The most general minisuperspace models analyzed in the literature correspond to homogeneous and anisotropic Bianchi type cosmological models. Since the corresponding metrics depend only on time, the dynamics of the spacelike 3-dimensional slices becomes trivial, unless an additional reparametrization is performed. Usually, in the reparametrization one of the scale factors of the Bianchi type metric, i.e., the volume $\Omega$ of the Misner parametrization, is fixed as internal time, as consequence of fixing a gauge, so that the Wheeler-DeWitt equation generates a state function of the universe which explicitly depends on the gauge fixed internal time and on the remaining scale factors, related to the anisotropy of such models. It is worthwhile to stress that the volume $\Omega$ is not a proper time parameter.

It has been found that in the framework of the minisuperspace sector of simple supergravity approach, without having a (super) Casimir operator, there are no physical states. Moreover, there exists only a non-physical trivial rest frame type (bosonic state) state function [14]. However, the trivial "rest frame" type solution exist only for arbitrary Lorentz symmetry generators [15, 16, 17, 18, 19, 20, 21, 22].

In all the cases the failure to find physical states [23] could be attributed to the fact that, due to the strong symmetry reduction, only a finite number of degrees of freedom can be considered, in the minisuperspace. To face this difficulty one needs to analyze genuine field theories with an infinite number of degrees of freedom. An option would be to consider milder symmetry reductions which leave unaffected a specific set of true local degrees of freedom. These are the so called midisuperspace models, which break the homogeneity of 
the standard Bianchi models. The midisuperspace models provide a canonical description of Einstein spacetimes with a group of isometries. Symmetries remove infinitely many degrees of freedom of the gravitational field, but there still remain infinitely many degrees of freedom. In spite of this simplification, the midisuperspace constraints of general relativity are still complicated functionals of the canonical variables, without a natural time parameter.

The simplest midisuperspace generalization of the homogeneous minisuperspace models are the Gowdy cosmological models, since they possess two Killing vectors, i.e., two ignorable coordinates, reducing the problem to time (as in standard quantum cosmology) and to one space coordinate, which completely eliminates homogeneity and leads to a system with an infinite number of degrees of freedom, i.e., a true field theory. Such spacetimes have a long history in general relativity. The field equations in this case can be shown to be equivalent to the wave equation for a scalar field propagating in a fictitious flat $(2+1)$-dimensional spacetime [24]. The local degrees of freedom are contained in the scalar field. In fact, the study of midisuperspace models and covariant field systems like string models indicates that if there exists an internal time which converts the old constraints of general relativity into a Schrödinger equation form, such a time variable is non-local functional of the geometric variables.

The Gowdy $T^{3}$ cosmological models have been analyzed in the context of non-perturbative canonical quantization of gravity [25, 26]. The arbitrariness in the selection of a time parameter is a problem that immediately appears in the process of quantization. For a specific choice of time, it was shown that there does not exist a unitary operator that could be used to generate the corresponding quantum evolution. Therefore, even in the case of midisuperspace models there is no natural time parameter.

In this work we will consider the specific midisuperspace described by Gowdy $T^{3}$ cosmological models [27, 28], in the context of $N=1$ supergravity. The quantum constraints of the theory are analyzed in the search of physical states.

This paper is organized as follows. In Section II, the canonical formulation of simple supergravity $N=1$ is briefly revisited. In [II, the model independent Lorentz constraint is analyzed and explicitly solved, following closely notations and conventions of [23]. In Section IV, the Gowdy $T^{3}$ cosmological models and their main properties are reviewed. Section $\mathrm{V}$ is devoted to the investigation of the model dependent supersymmetric constraint, and in VI we find solutions for the state function of the universe, for both the polarized case and 
for the unpolarized case. The last section contains several final remarks.

\section{CANONICAL FORMULATION OF $N=1$ SUPERGRAVITY}

The starting point is the $(N=1)$ supergravity Lagrangian

$$
\mathcal{L}=\frac{1}{2} \sqrt{-g} R-\frac{i}{2} \varepsilon^{\lambda \mu \nu \rho} \overline{\Psi_{\lambda}} \gamma_{5} \gamma_{\mu} D_{\nu} \Psi_{\rho}
$$

where

$$
D_{\nu}=\partial_{\nu}+(1 / 2) \omega_{\nu A B} \sigma^{A B}
$$

is the covariant derivative and $\sigma^{A B}:=(1 / 4)\left(\gamma^{A} \gamma^{B}-\gamma^{B} \gamma^{A}\right)$.

For the $\gamma^{A}$ matrices we use a real Majorana representation

$$
\gamma^{0}=\left(\begin{array}{cc}
0 & \sigma^{2} \\
\sigma^{2} & 0
\end{array}\right), \gamma^{1}=\left(\begin{array}{cc}
i \sigma^{3} & 0 \\
0 & i \sigma^{3}
\end{array}\right), \gamma^{2}=\left(\begin{array}{cc}
0 & -\sigma^{2} \\
\sigma^{2} & 0
\end{array}\right), \gamma^{3}=\left(\begin{array}{cc}
-i \sigma^{1} & 0 \\
0 & -i \sigma^{1}
\end{array}\right)
$$

in which the anticommutator relation $\left\{\gamma^{A}, \gamma^{B}\right\}=2 \eta^{A B}$ is satisfied, and $\sigma^{i}$ are the standard Pauli matrices. Moreover, $\gamma_{5}=i \gamma^{0} \gamma^{1} \gamma^{2} \gamma^{3}$. The Rarita-Schwinger field $\Psi:=\Psi_{A} \omega^{A}$, a spinor-valued one-form, is subject to the Majorana condition $\bar{\Psi}=\Psi^{T} C$, with $C$ the charge conjugation matrix. The vector-spinor gravitino field can be written in components form as

$$
\Psi_{\mu \mathcal{A}}=\left(\begin{array}{c}
\psi_{\mu 1} \\
\psi_{\mu 2} \\
\psi_{\mu 3} \\
\psi_{\mu 4}
\end{array}\right)
$$

where $\mu$ is a vector index and $\mathcal{A}$ is a spinor index. In this representation the Majorana condition reads $\bar{\Psi}=-i \Psi^{T} \gamma^{0}$.

The coupling constant is set to one and the Ricci rotation coefficients $\omega_{\nu A B}$ read

$$
\omega_{\nu A B}=\widetilde{\omega}_{\nu A B}+K_{\nu A B}
$$

where $\widetilde{\omega}_{\nu A B}$ are the standard Levi-Civita Ricci rotation coefficients. The contortion tensor is

$$
K_{\nu A B}=e_{A}^{\mu} e_{B}^{\rho} K_{\nu \mu \rho} \quad \text { and } \quad K_{\nu \mu \rho}=\frac{1}{2}\left(T_{\nu \mu \rho}-T_{\mu \rho \nu}+T_{\rho \nu \mu}\right) .
$$

Greek indices from the end of the alphabet, i.e. $\lambda, \mu, \nu, \rho, \cdots$, always range over $0,1,2,3$, Greek indices from the beginning of the alphabet i.e. $\alpha, \beta, \gamma, \cdots$, over $1,2,3$, and both refer 
to world coordinates. Capital Latin indices, i.e. $A, B, \ldots$ run over $0,1,2,3$ and small Latin indices, i.e. $a, b, \ldots$ over $1,2,3$, and are those with respect to a local orthonormal basis.

In the case at hand the canonical variables are the covariant spatial components of the vierbein $e^{a}{ }_{\alpha}$, their conjugate momenta $p_{a}{ }^{\alpha}$, and the spatial covariant components of the vector spinor $\Psi_{\alpha}$, defined on a generic spacelike hypersurface. There are three different constraints in the problem, namely, the generators $\mathcal{H}_{\mu}$ of the translations and diffeomorphisms, the generators $\mathcal{J}_{\mu \nu}$ of local Lorentz rotations and the Majorana spinor supersymmetric generators $\mathcal{S}$.

The Lagrange multipliers constraining the generators of translations, rotations, and supersymmetry transformations are the normal components $e^{A}{ }_{0}, \omega_{0 A B}$, and $\bar{\Psi}_{0}$, respectively, of the corresponding gauge fields $e^{A}{ }_{\mu}, \omega_{\mu A B}$ and $\bar{\Psi}_{\mu}$ with respect to the timelike normal vector $\mathbf{n}$.

Therefore, the canonical form of the simple $(N=1)$ supergravity Lagrangian (11) can be written as [7]

$$
\begin{aligned}
H & =e^{A}{ }_{0} \mathcal{H}_{A}+\frac{1}{2} \omega_{0}{ }^{A B} \mathcal{J}_{A B}+\bar{\Psi}_{0} \mathcal{S} \\
& =N \mathcal{H}_{\perp}+N^{i} \mathcal{H}_{i}+\frac{1}{2} \omega_{0 A B} \mathcal{J}^{A B}+\bar{\Psi}_{0} \mathcal{S}
\end{aligned}
$$

where $\mathcal{H}_{A}, \mathcal{J}_{A B}$ and $\mathcal{S}$ are constructed from the canonical variables only and do not depend on the multipliers. In the equivalent form of the canonical Lagrangian $\mathcal{H}_{\perp}, \mathcal{H}_{i}$ and $\mathcal{J}^{A B}$ are the usual Hamiltonian, diffeomorphism, and rotational Lorentz bosonic constraints, respectively, and $\mathcal{S}$ the supersymmetric fermionic constraint. Now the lapse function $N=e_{0}{ }^{0}$, the shift vector $N_{i}=e_{i}{ }^{0}, \omega_{0 A B}$, and $\bar{\Psi}_{0}$ are the corresponding Lagrange multipliers. The supergravity generators satisfy the following algebra discovered by Teitelboim [5]:

$$
\begin{aligned}
\left\{\mathcal{S}(x), \overline{\mathcal{S}}\left(x^{\prime}\right)\right\} & =\gamma^{A} \mathcal{H}_{A} \delta\left(x, x^{\prime}\right), \\
{\left[\mathcal{S}(x), \mathcal{H}_{C}\left(x^{\prime}\right)\right] } & =\frac{1}{2} \Sigma_{C A B} \mathcal{J}^{A B} \delta\left(x, x^{\prime}\right), \\
{\left[\mathcal{S}(x), \mathcal{J}^{A B}\left(x^{\prime}\right)\right] } & =-\sigma^{A B} \mathcal{S} \delta\left(x, x^{\prime}\right), \\
{\left[\mathcal{H}_{A}(x), \mathcal{H}_{B}\left(x^{\prime}\right)\right] } & =\left(-T_{A B}{ }^{C} \mathcal{H}_{C}+\frac{1}{2} \Omega_{A B C D} \mathcal{J}^{C D}+\bar{H}_{A B} \mathcal{S}\right) \delta\left(x, x^{\prime}\right), \\
{\left[\mathcal{H}_{C}(x), \mathcal{J}^{A B}\left(x^{\prime}\right)\right] } & =\left(\delta_{C}^{B} \mathcal{H}^{A}-\delta_{C}^{A} \mathcal{H}^{B}\right) \delta\left(x, x^{\prime}\right), \\
{\left[\mathcal{J}^{A B}(x), \mathcal{J}^{C D}\left(x^{\prime}\right)\right] } & =\left(\eta^{A C} \mathcal{J}^{B D}-\eta^{B C} \mathcal{J}^{A D}+\eta^{B D} \mathcal{J}^{A C}-\eta^{A D} \mathcal{J}^{B C}\right) \delta\left(x, x^{\prime}\right) .
\end{aligned}
$$

Note that even the bosonic part is only a closed soft gauge algebra [29] due to the appearance 
of torsion and curvature, instead of structure constants, on the right hand side. The fields

$$
\begin{aligned}
H_{A B} & =D_{A} \Psi_{B}-D_{B} \Psi_{A}, \\
\Sigma_{A B C} & =\gamma_{5}\left(\gamma_{A}^{*} H_{B C}+\frac{1}{2} e_{A}{ }^{\mu} e_{[B \mu} \gamma_{D}{ }^{*} H_{C]}{ }^{D}\right), \\
\Omega_{A B C D} & =R_{A B C D}-\bar{\Psi}_{[A} \Sigma_{B] C D},
\end{aligned}
$$

play the role of curvature two-forms and depend on the canonical variables of the theory. Without them, the algebra goes over into the supersymmetry algebra of flat space [30].

Consequently, physical states $|\Psi\rangle$ in the quantum theory have to satisfy the conditions

$$
\mathcal{S}|\Psi\rangle=0, \quad \mathcal{H}_{A}|\Psi\rangle=0, \quad \mathcal{J}_{A B}|\Psi\rangle=0
$$

Note that the supersymmetric constraint $\mathcal{S}|\Psi\rangle=0$ is the "square root" of the Hamiltonian one, on account of (8), and implies $\mathcal{H}_{A}|\Psi\rangle=0$, so the second condition is redundant. Thus, we will focus only on the Lorentz $\mathcal{J}_{A B}$ and supersymmetric $\mathcal{S}$ constraints, which are explicitly given as follows [7]:

$$
\begin{aligned}
\mathcal{J}_{A B} & \equiv p_{A}{ }^{\alpha} e_{B \alpha}-p_{B}{ }^{\alpha} e_{A \alpha}-\pi^{\alpha}{ }_{\mathcal{A}} \sigma_{A B} \Psi_{\alpha}{ }^{\mathcal{A}} \\
& =2 p_{[A}{ }^{\alpha} e_{B] \alpha}+\tau_{A B 0} \\
& =2 p_{[A}{ }^{\alpha} e_{B] \alpha}+\frac{1}{2} \phi_{[A \mathcal{A}}^{T} \phi_{B]}{ }^{\mathcal{A}}
\end{aligned}
$$

where

$$
\tau_{\mu \nu \lambda}=\frac{i}{4} \bar{\Psi}_{[\mu \mid} \gamma_{\lambda} \Psi_{\mid \nu]}
$$

are the components of the spin tensor, see (8.7) of [31], $\phi_{A \mathcal{A}}$ are the desitized local gravitino components (see Eq. (25)), and

$$
\pi^{\alpha}=\frac{i}{2} \varepsilon^{0 \alpha \delta \beta} \bar{\Psi}_{\delta} \gamma_{5} \gamma_{\beta}
$$

is the momentum conjugate to the gravitino field. In the last step we have used the Majorana condition [32] $\bar{\Psi}=\Psi^{T} C=-i \Psi^{T} \gamma^{0}$. Equivalently in terms of the dual generators

$$
\mathcal{J}_{A}=\frac{i}{2} \epsilon_{0 A B C} \mathcal{J}^{B C} \quad \Rightarrow \quad \mathcal{J}_{0}=0
$$

the Lorentz constraint reads

$$
\mathcal{J}_{A}=\frac{i}{2} \epsilon_{0 A B C}\left[2 p^{[B \alpha} e^{C]}{ }_{\alpha}+\frac{1}{2} \phi^{T[B}{ }_{\mathcal{A}} \phi^{C] \mathcal{A}}\right] .
$$


It is interesting to note that, as expected due to the time arbitrariness, the condition $\mathcal{J}_{0}=0$ implies that $\mathcal{J}_{0 B} \equiv 0$, therefore reducing the Lorentz constraint to pure spatial rotations on the hypersurface of constant time.

The generator of supersymmetry reads [7]

$$
\mathcal{S}=-i \epsilon^{i j k} \gamma_{5} \gamma_{i} \nabla_{j} \Psi_{k}-\frac{i}{2} p^{\alpha}{ }_{A} \gamma^{A} \Psi_{\alpha}+\frac{1}{4}{ }^{(3)} e \gamma_{\perp} \psi_{\alpha} \bar{\psi}^{\alpha} \gamma^{\beta} \Psi_{\beta},
$$

where $\gamma_{\perp}=-N \gamma^{0}$, with $N$ the lapse function.

A further constraint, the Cartan relation

$$
T_{\mu \nu \lambda}=-4 \tau_{\mu \nu \lambda}=-i \bar{\Psi}_{[\mu \mid} \gamma_{\lambda} \Psi_{\mid \nu]},
$$

relates the torsion tensor to the Rarita-Schwinger field and is used to eliminate the torsion from the theory, leaving it only with first class constraints [34].

It is rather convenient to use instead of the gravitino field itself, its densitized local components

$$
\phi_{a}=e e_{a}^{\alpha} \Psi_{\alpha}
$$

as the basic fields commuting with all non-spinor variables, here $e={ }^{(3)} e=\operatorname{det}\left(e_{a}^{\alpha}\right)$. This variable was already found to be the natural one for the gravitino field, see [33]. This choice suggests a matrix realization of the $\phi_{i \mathcal{A}}$ obeying

$$
\left\{\phi_{i \mathcal{A}}, \phi_{j \mathcal{B}}\right\}=-\frac{i}{8}\left(\gamma_{j} \gamma_{i}\right)_{\mathcal{A B}} .
$$

Here $\mathcal{A}$ and $\mathcal{B}$ are spinor indices, and the gravitational variables appear nowhere.

\section{LORENTZ CONSTRAINT}

We will assume the following form for the wave function of the universe

$$
|\Psi\rangle=\Psi_{\mu}=\left(\begin{array}{c}
\Psi_{I} \\
\Psi_{I I} \\
\Psi_{I I I} \\
\Psi_{I V}
\end{array}\right),
$$

Using the real Majorana representation (3) for the $\gamma$-matrices [14, 35] as well as the anticommuting relation (26) between the components of the gravitino field, we can write the components of the Lorentz generator (22) and of the supersymmetric generator (23). 
It is well known that as we fix a particular basis for the vierbein, as, for instance, the $S O(3)$ one, the Lorentz constraint (22) reduces to

$$
\mathcal{J}_{A}=\frac{i}{2} \epsilon_{0 A B C}\left[\frac{i}{2} \phi_{[B \mathcal{A}}^{T} \phi_{C]} \mathcal{A}\right] \text {. }
$$

Therefore

$$
\begin{aligned}
\mathcal{J}_{1} & =-\frac{i}{2}\left[\phi_{2 \mathcal{A}} \phi_{3}{ }^{\mathcal{A}}+\phi_{3 \mathcal{A}} \phi_{2}{ }^{\mathcal{A}}\right] \\
\mathcal{J}_{2} & =-\frac{i}{2}\left[\phi_{3 \mathcal{A}} \phi_{1}{ }^{\mathcal{A}}+\phi_{1 \mathcal{A}} \phi_{3}{ }^{\mathcal{A}}\right], \\
\mathcal{J}_{3} & =-\frac{i}{2}\left[\phi_{1 \mathcal{A}} \phi_{2}{ }^{\mathcal{A}}+\phi_{2 \mathcal{A}} \phi_{1}{ }^{\mathcal{A}}\right] .
\end{aligned}
$$

By means of the algebra (26), which the components of the gravitino field fulfill, we arrive at a realization of the components of the Lorentz constraint in terms of the standard generators of the ordinary rotation group $O(3)[14]$

$$
\mathcal{J}_{3}=-i\left(\begin{array}{cccc}
0 & 0 & 0 & 0 \\
0 & 0 & 1 & 0 \\
0 & -1 & 0 & 0 \\
0 & 0 & 0 & 0
\end{array}\right), \mathcal{J}_{2}=-i\left(\begin{array}{cccc}
0 & 0 & 0 & 0 \\
0 & 0 & 0 & -1 \\
0 & 0 & 0 & 0 \\
0 & 1 & 0 & 0
\end{array}\right), \mathcal{J}_{1}=-i\left(\begin{array}{cccc}
0 & 0 & 0 & 0 \\
0 & 0 & 0 & 0 \\
0 & 0 & 0 & 1 \\
0 & 0 & -1 & 0
\end{array}\right) \text {. }
$$

Consequently, for instance the component $\Psi_{I I}$ of the state function should have four components, i.e., $\Psi_{I I}=\left(\Psi_{I I}^{1}, \Psi_{I I}^{2}, \Psi_{I I}^{3}, \Psi_{I I}^{4}\right)$, analogously for $\Psi_{I I I}$, and $\Psi_{I V}$.

Let us analyze the Lorentz condition $\mathcal{J}_{A B}|\Psi\rangle=0$ which explicitly reads

$$
\mathcal{J}_{A B}|\Psi\rangle=\left(\begin{array}{cccc}
0 & 0 & 0 & 0 \\
0 & 0 & \mathcal{J}_{12} & \mathcal{J}_{13} \\
0 & -\mathcal{J}_{12} & 0 & \mathcal{J}_{23} \\
0 & -\mathcal{J}_{13} & -\mathcal{J}_{23} & 0
\end{array}\right)\left(\begin{array}{c}
\Psi_{I} \\
\Psi_{I I} \\
\Psi_{I I I} \\
\Psi_{I V}
\end{array}\right)=0
$$

This implies the conditions [42]

$$
\begin{aligned}
\mathcal{J}_{12} \Psi_{I I I} & =-\mathcal{J}_{13} \Psi_{I V} \\
\mathcal{J}_{12} \Psi_{I I} & =\mathcal{J}_{23} \Psi_{I V} \\
\mathcal{J}_{13} \Psi_{I I} & =-\mathcal{J}_{23} \Psi_{I I I}
\end{aligned}
$$

or equivalently, we can write the conditions (34)-(36) as

$$
\begin{aligned}
\mathcal{J}_{3} \Psi_{I I I} & =\mathcal{J}_{2} \Psi_{I V} \\
\mathcal{J}_{3} \Psi_{I I} & =\mathcal{J}_{1} \Psi_{I V} \\
\mathcal{J}_{2} \Psi_{I I} & =\mathcal{J}_{1} \Psi_{I I I}
\end{aligned}
$$


respectively.

It is interesting to note that there is no condition in (34)-(36) or equivalently in (37)(39) involving $\Psi_{I}$. By replacing the representation (32) into Eqs. (37)-(39), one obtains the following system of algebraic equations for the different components of the state function of the universe

$$
\begin{array}{ll}
\Psi_{I I I}^{2}=\Psi_{I V}^{2}=0, & \Psi_{I I I}^{3}=-\Psi_{I V}^{4} \\
\Psi_{I I}^{3}=\Psi_{I V}^{3}=0, & \Psi_{I I}^{2}=-\Psi_{I V}^{4} \\
\Psi_{I I}^{4}=\Psi_{I I I}^{4}=0, & \Psi_{I I}^{2}=-\Psi_{I I I}^{3} .
\end{array}
$$

The solution of (40)-(42) is straightforward and reads

$$
|\Psi\rangle=\left(\begin{array}{c}
\Psi_{I} \\
\Psi_{I I}^{1} \\
\Psi_{I I I}^{1} \\
\Psi_{I V}^{1}
\end{array}\right)
$$

and reduces each of the $\Psi_{I I}, \Psi_{I I I}$, and $\Psi_{I V}$ to only one component.

This ends the analysis of the Lorentz constraint. Notice that in the bosonic Wigner "rest-frame"-like solution for the state function of the universe is a scalar with only one independent component [23].

\section{GOWDY $T^{3}$ COSMOLOGICAL MODELS}

Gowdy cosmological models are inhomogeneous time-dependent solutions of Einstein's vacuum equations with compact Cauchy spatial hypersurfaces whose topology can be either $T^{3}$ or $S^{1} \times S^{2}$ [27, 28]. Other particular topologies are contained in these two as special cases. Here we will focus on $T^{3}$ models for which the line element can be written as [36]

$$
d s^{2}=e^{-\frac{\lambda}{2}+3 \tau} d \tau^{2}-e^{-\frac{\lambda}{2}-\tau} d \chi^{2}-e^{2 \tau}\left[e^{P}(d \sigma+Q d \delta)^{2}+e^{-P} d \delta^{2}\right]
$$

where $P, Q, \lambda$, and $\tau$ depend on the non-ignorable coordinates $t$ and $\chi$. The spatial hypersurfaces $(\tau=$ const. $)$ are compact if we require that $0 \leq \chi, \sigma, \delta \leq 2 \pi$. The expression in square brackets depicts the metric on the $T^{2}$ subspace which is generated by the commuting Killing vectors $\partial_{\sigma}$ and $\partial_{\delta}$. The coordinate $\chi$ labels the different tori. 
When the Killing vectors are hypersurface orthogonal, the general line element (44) becomes diagonal with $Q=0$ and the corresponding cosmological models are called polarized. In this last case, the subspace $T^{2}$ corresponds to the spatial surfaces of a $(2+1)$ fictitious flat spacetime in which a scalar field, represented by the metric structural function $P$, propagates [25]. The local degrees of freedom contained in the scalar field are true gravitational degrees of freedom which cannot be eliminated by a choice of gauge. We are thus facing a genuine field theory which is a special case of a midisuperspace model. Notice that the infinite number of degrees of freedom contained in this midisuperspace model can be associated with the inhomogeneous character of the spacetime. If we neglect the inhomogeneities present in the model, we would obtain a minisuperspace model with a finite number of degrees of freedom, probably related to a Bianchi cosmological model. The general unpolarized case $(Q \neq 0)$ also corresponds to a midisuperspace model; however, its interpretation in terms of a dynamical scalar field in a $(2+1)$ spacetime can not be realized.

In order to write the Gowdy line element (44) in ADM form [37] we introduce the lapse $N$ and shift functions $N_{i}$ as follows, c.f. [36]

$$
\begin{aligned}
& N=g^{-1 / 2}\left[g^{00}\right]^{-1 / 2}=\exp \left[\frac{1}{4} \lambda-\frac{3}{2} \tau\right]\left[g^{00}\right]^{-1 / 2}, \\
& N_{i}=g_{0 i}=0,
\end{aligned}
$$

where, as usual, $N$ and $N_{i}$ are gauge functions usually fixed to $N=1$, and $N_{i}=0$, which implies a restriction on the time development of the coordinates off the initial hypersurface. A further restriction is that $\tau$ does not depend on $\chi$, i.e.,

$$
\frac{\partial \tau}{\partial \chi}=0, \quad \Rightarrow \quad \tau=\tau(t), \quad \Rightarrow \quad \lambda=\lambda(t)
$$

is also introduced in order to reduce the configuration space of the problem to one in which $\lambda$ has only one degree of freedom, i.e., $\lambda=\lambda(t)$, although $P$ and $Q$ retain their infinitely many degrees of freedom as arbitrary functions of $\chi[38]$.

Therefore, Eq. (44) can be written as

$$
d s^{2}=N^{2} d \tau^{2}-e^{-\frac{\lambda}{2}-\tau} d \chi^{2}-e^{2 \tau}\left[e^{P}(d \sigma+Q d \delta)^{2}+e^{-P} d \delta^{2}\right]
$$

The structure of the line element (48) suggests the following choice for the basis

$$
\omega^{0}=d \tau, \quad \omega^{1}=d \chi, \quad \omega^{2}=(d \sigma+Q d \delta), \quad \omega^{3}=d \delta
$$


in order to write the Gowdy line element in the standard ADM form, i.e., $d s^{2}=N^{2} d \tau^{2}+$ $g_{i j} \omega^{i} \omega^{j}[2]$. Therefore, in this basis the metric (48) reduces

$$
d s^{2}=N^{2} d \tau^{2}-e^{\left(-\frac{\lambda}{2}-\tau\right)}\left(\omega^{1}\right)^{2}-e^{2 \tau}\left[e^{P}\left(\omega^{2}\right)^{2}+e^{-P}\left(\omega^{3}\right)^{2}\right]
$$

hence, the corresponding coframe reads

$$
e^{0}=N d \tau, \quad e^{1}=e^{\left(-\frac{\lambda}{4}-\frac{\tau}{2}\right)} \omega^{1}, \quad e^{2}=e^{\left(\tau+\frac{P}{2}\right)} \omega^{2}, \quad e^{3}=e^{\left(\tau-\frac{P}{2}\right)} \omega^{3},
$$

and satisfies the standard orthonormality condition $g^{\mu \nu} e^{A}{ }_{\mu} e^{B}{ }_{\nu}=\eta^{A B}$, with $e^{A}=e_{\mu}^{A} \omega^{\mu}$. The dual basis to the coframe (51) reads

$$
\Omega_{0}=N^{-1} \partial_{\tau}, \quad \Omega_{1}=e^{\left(\frac{\lambda}{4}+\frac{\tau}{2}\right)} \omega_{1}, \quad \Omega_{2}=\frac{1}{2} e^{\left(-\tau-\frac{P}{2}\right)} \omega_{2}, \quad \Omega_{3}=e^{\left(-\tau+\frac{P}{2}\right)} \omega_{3},
$$

where $\omega_{1}=\partial_{\chi}, \omega_{2}=\partial_{\delta}$, and $\omega_{3}=-Q \partial_{\sigma}+\partial_{\delta}$ are the components of the dual basis to (49). In the basis (51) it is straightforward to calculate the connection one-form, i.e., $d e^{A}=-\omega^{A}{ }_{C} \wedge e^{C}=-\omega^{A}{ }_{B C} e^{B} \wedge e^{C}$. Hence, the only non-vanishing components of the connection $\omega_{A B C}$ read

$$
\begin{aligned}
& \omega_{110}=-\omega_{101}=\frac{1}{2}\left[\frac{\dot{\lambda}}{2}+\dot{\tau}\right], \quad \omega_{220}=-\omega_{202}=-\left[\frac{\dot{P}}{2}+\dot{\tau}\right], \quad \omega_{230}=-\omega_{203}=-e^{P} \dot{Q}, \\
& \omega_{221}=-\omega_{212}=-e^{\frac{\lambda}{4}+\frac{\tau}{2}} \frac{P_{\chi}}{2}, \quad \omega_{231}=-\omega_{213}=-e^{\frac{\lambda}{4}+\frac{\tau}{2}} e^{P} Q_{\chi}, \quad \omega_{330}=-\omega_{303}=\frac{\dot{P}}{2}-\dot{\tau}, \\
& \omega_{331}=-\omega_{313}=e^{\frac{\lambda}{4}+\frac{\tau}{2}} \frac{P_{\chi}}{2},
\end{aligned}
$$

where the dot means time derivative. Therefore, the corresponding covariant derivative, i.e., $\nabla_{a}=\Omega_{a}+\frac{1}{4} \omega_{a b c} \gamma^{b} \gamma^{c}$, reads

$$
\begin{aligned}
\nabla_{1} & =e^{\frac{\lambda}{4}+\frac{\tau}{2}} \omega_{1}+\frac{1}{4}\left[\frac{\dot{\lambda}}{2}+\dot{\tau}\right] \gamma^{1} \gamma^{0} \\
\nabla_{2} & =\frac{1}{2} e^{(-\tau-P / 2)} \omega_{2}-\frac{1}{2}\left[\frac{\dot{P}}{2}+\dot{\tau}\right] \gamma^{2} \gamma^{0}-\frac{1}{2} e^{P} \dot{Q} \gamma^{3} \gamma^{0}-e^{\frac{\lambda}{4}+\frac{\tau}{2}} \frac{P_{\chi}}{4} \gamma^{2} \gamma^{1} \\
& -\frac{1}{2} e^{\frac{\lambda}{4}+\frac{\tau}{2}} e^{P} Q_{\chi} \gamma^{3} \gamma^{1} \\
\nabla_{3} & =e^{(-\tau+P / 2)} \omega_{3}+\frac{1}{2}\left[\frac{\dot{P}}{2}-\dot{\tau}\right] \gamma^{3} \gamma^{0}+e^{\frac{\lambda}{4}+\frac{\tau}{2}} \frac{P}{4} \gamma^{3} \gamma^{1}
\end{aligned}
$$

According to (25), the densitized local components of the gravitino field are thus given by

$$
\psi_{1}=e^{(-2 \tau)} \phi_{1}, \quad \psi_{2}=e^{\left(\frac{\lambda}{4}-\frac{\tau}{2}+\frac{P}{2}\right)} \phi_{2}, \quad \psi_{3}=e^{\left(\frac{\lambda}{4}-\frac{\tau}{2}-\frac{P}{2}\right)} \phi_{3}
$$




\section{SUPERSYMMETRIC CONSTRAINT}

Since we are considering simple $(N=1)$ supergravity, i.e., only two supersymmetric charges, whose square vanishes, the general expression (23) for the supersymmetric constraint reduces to

$$
\begin{aligned}
\mathcal{S} & =-i \epsilon^{a b c} \gamma_{5} \gamma_{a} \nabla_{b} e e_{c}{ }^{k} \Psi_{k}-\frac{i}{2} p^{\alpha}{ }_{A} \gamma^{A} e^{-1} e^{a}{ }_{\alpha} \phi_{a} \\
& =i\left\{\left(\gamma^{1} \phi_{1}-\gamma^{3} \phi_{3}\right) \frac{\Pi_{\tau}}{4}-\left(\gamma^{2} \phi_{2}+\gamma^{3} \phi_{3}-\gamma^{1} \phi_{1}\right) \frac{\Pi_{\lambda}}{8}-\left(3 \gamma^{2} \phi_{2}-2 \gamma^{3} \phi_{3}\right) \frac{\Pi_{P}}{4}\right. \\
& -\left(\gamma^{2} \phi_{3}-\gamma^{1} \gamma^{2} \gamma^{3} \phi_{1}\right) e^{P} \frac{\Pi_{Q}}{2}-\gamma^{0} \gamma^{1}\left(\gamma^{3} \phi_{3}-\gamma^{2} \phi_{2}\right) e^{\left(-\frac{\lambda}{2}+\frac{\tau}{2}\right)} \frac{P_{\chi}}{4} \\
& \left.+\gamma^{0} \gamma^{2}\left(\gamma^{3} \phi_{1}-\gamma^{1} \phi_{3}\right) e^{\left(-\frac{\lambda}{2}+\frac{\tau}{2}\right)} e^{P} \frac{Q_{\chi}}{2}\right\},
\end{aligned}
$$

where $\Pi_{\tau}, \Pi_{\lambda}, \Pi_{P}$, and $\Pi_{Q}$ are the conjugated momenta, in the selected foliation, to $\tau, \lambda$, $P$, and $Q$, respectively, and the subindex $\chi$ means $\frac{d}{d \chi}$.

The complete supersymmetric constraint is obtained by integrating the $\chi$-dependence in (58), i.e.,

$$
\mathfrak{S}=\int_{0}^{2 \pi} \mathcal{S} d \chi .
$$

In order to perform the integration, we expand our generalized coordinates and their conjugated momenta in terms of the one-dimensional complete set of functions $(\cos m \chi, \sin m \chi)$, namely,

$$
\begin{aligned}
\Pi_{P} & =\Pi_{P 0}+\sum_{n=1}^{\infty}\left(\Pi_{P n} \cos n \chi+\Pi_{P-n} \sin n \chi\right) \\
P & =P_{0}+\sum_{n=1}^{\infty}\left(P_{n} \cos n \chi+P_{-n} \sin n \chi\right) \\
\Pi_{Q} & =\Pi_{Q 0}+\sum_{n=1}^{\infty}\left(\Pi_{Q n} \cos n \chi+\Pi_{Q-n} \sin n \chi\right) \\
Q & =Q_{0}+\sum_{n=1}^{\infty}\left(Q_{n} \cos n \chi+Q_{-n} \sin n \chi\right),
\end{aligned}
$$

this implies that

$$
\begin{aligned}
& \int_{0}^{2 \pi} \Pi_{P} d \chi=\int_{0}^{2 \pi}\left[\Pi_{P 0}+\sum_{n=1}^{\infty}\left(\Pi_{P n} \cos n \chi+\Pi_{P-n} \sin n \chi\right)\right] d \chi=2 \pi \Pi_{P 0} \\
& \int_{0}^{2 \pi} P_{\chi} d \chi=\left.P\right|_{0} ^{2 \pi}=\left.\left[P_{0}+\sum_{n=1}^{\infty}\left(P_{n} \cos n \chi+P_{-n} \sin n \chi\right)\right]\right|_{0} ^{2 \pi}=P_{0} .
\end{aligned}
$$


Therefore, assuming the condition (65) for $P$,

$$
\begin{aligned}
& \int_{0}^{2 \pi} e^{P} \Pi_{Q} d \chi=e^{P_{0}} \int_{0}^{2 \pi}\left[\Pi_{Q 0}+\sum_{n=1}^{\infty}\left(\Pi_{Q n} \cos n \chi+\Pi_{Q-n} \sin n \chi\right)\right] d \chi=2 \pi e^{P_{0}} \Pi_{Q 0} \\
& \int_{0}^{2 \pi} e^{P} Q_{\chi} d \chi=\left.e^{P_{0}} Q\right|_{0} ^{2 \pi}=\left.e^{P_{0}}\left[Q_{0}+\sum_{n=1}^{\infty}\left(Q_{n} \cos n \chi+Q_{-n} \sin n \chi\right)\right]\right|_{0} ^{2 \pi}=e^{P_{0}} Q_{0}
\end{aligned}
$$

Hence, the final form of the supersymmetric constraint (158) reads

$$
\begin{aligned}
\mathfrak{S} & =\frac{i}{4}\left\{\left(\gamma^{1} \phi_{1}-\gamma^{3} \phi_{3}\right) \Pi_{\tau}-\left(\gamma^{2} \phi_{2}+\gamma^{3} \phi_{3}-\gamma^{1} \phi_{1}\right) \frac{\Pi_{\lambda}}{2}-2\left(3 \gamma^{2} \phi_{2}-2 \gamma^{3} \phi_{3}\right) \Pi_{P_{0}}\right. \\
& -4\left(\gamma^{2} \phi_{3}-\gamma^{1} \gamma^{2} \gamma^{3} \phi_{1}\right) e^{P_{0}} \Pi_{Q_{0}}-\gamma^{0} \gamma^{1}\left(\gamma^{3} \phi_{3}-\gamma^{2} \phi_{2}\right) e^{\left(-\frac{\lambda}{2}+\frac{\tau}{2}\right)} P_{0} \\
& \left.-2 \gamma^{0} \gamma^{2}\left(\gamma^{3} \phi_{1}-\gamma^{1} \phi_{3}\right) e^{\left(-\frac{\lambda}{2}+\frac{\tau}{2}\right)} e^{P_{0}} Q_{0}\right\},
\end{aligned}
$$

where a constant factor has been included in a redefinition of all integrated quantities.

\section{PHYSICAL STATES}

In order to quantize the problem that we have outlined above, we will convert $\Pi_{\tau}, \Pi_{\lambda}$, $\Pi_{P_{0}}, \Pi_{Q_{0}}, P_{0}$, and $Q_{0}$ into operators $i \frac{\delta}{\delta \tau}, i \frac{\delta}{\delta \lambda}, i \frac{\delta}{\delta P_{0}}, \frac{\delta}{\delta Q_{0}}, \widehat{P}_{0}, \widehat{Q}_{0}$, respectively. They act on the state function of the universe $\Psi$ and the supersymmetric constraint $\mathfrak{S}$, Eq. ([68), becomes also an operator which, according to the Dirac canonical quantization procedure, should annihilate the state function of the universe, i.e.,

$$
\widehat{\mathfrak{S}}|\Psi\rangle=0
$$

The solutions to the supersymmetric constraint, Eq. (69), for the state function $\Psi$ given by (43), as result of solving the Lorentz constraint, are known as physical states of the theory.

Since $\left\{\widehat{\mathfrak{S}}_{\mathcal{A}}, \widehat{\mathfrak{S}}_{\mathcal{B}}\right\}=0$, for $\mathcal{A} \neq \mathcal{B}$ we can take each $\widehat{\mathfrak{S}}_{\mathcal{A}}$ to operate in orthogonal subspaces, and we can write $\widehat{\mathfrak{S}}|\Psi\rangle=0$ in the form

$$
\left(\begin{array}{cccc}
\widehat{\mathfrak{S}}_{1} & 0 & 0 & 0 \\
0 & \widehat{\mathfrak{S}}_{2} & 0 & 0 \\
0 & 0 & \widehat{\mathfrak{S}}_{3} & 0 \\
0 & 0 & 0 & \widehat{\mathfrak{S}}_{4}
\end{array}\right)\left(\begin{array}{c}
\Psi_{I} \\
\Psi_{I I} \\
\Psi_{I I I} \\
\Psi_{I V}
\end{array}\right)=0
$$

where each of the $\widehat{\mathfrak{S}}_{\mathcal{A}}$ will be a matrix operator of the smallest rank possible that produces the appropriate algebra for $\widehat{\mathfrak{S}}$. 


\section{A. The polarized case $Q=0$}

The polarized case is obtained from (68) by setting the metric structure function $Q=0$, i.e.,

$$
\begin{aligned}
\widehat{\mathfrak{S}} & =\frac{i}{4}\left\{i\left(\gamma^{1} \phi_{1}-\gamma^{3} \phi_{3}\right) \frac{\delta}{\delta \tau}+\frac{i}{2}\left(\gamma^{2} \phi_{2}+\gamma^{3} \phi_{3}-\gamma^{1} \phi_{1}\right) \frac{\delta}{\delta \lambda}+2 i\left(3 \gamma^{2} \phi_{2}-2 \gamma^{3} \phi_{3}\right) \frac{\delta}{\delta P_{0}}\right. \\
& \left.-\gamma^{0} \gamma^{1}\left(\gamma^{3} \phi_{3}-\gamma^{2} \phi_{2}\right) e^{\left(-\frac{\lambda}{2}+\frac{\tau}{2}\right)} \widehat{P}_{0}\right\} .
\end{aligned}
$$

The operator $\widehat{\mathfrak{S}}$ has four spinor components:

$$
\begin{aligned}
\widehat{\mathfrak{S}}_{1} & =i\left(-\phi_{11}-\phi_{32}\right) \frac{\delta}{\delta \tau}+\frac{i}{2}\left(-\phi_{24}+\phi_{32}+\phi_{11}\right) \frac{\delta}{\delta \lambda}+2 i\left(-3 \phi_{24}-2 \phi_{32}\right) \frac{\delta}{\delta P_{0}} \\
& +\left(\phi_{21}+\phi_{33}\right) e^{\left(-\frac{\lambda}{2}+\frac{\tau}{2}\right)} \widehat{P}_{0}, \\
\widehat{\mathfrak{S}}_{2} & =i\left(\phi_{12}-\phi_{31}\right) \frac{\delta}{\delta \tau}+\frac{i}{2}\left(\phi_{23}+\phi_{31}-\phi_{12}\right) \frac{\delta}{\delta \lambda}+2 i\left(3 \phi_{23}-2 \phi_{31}\right) \frac{\delta}{\delta P_{0}} \\
& +\left(-\phi_{22}+\phi_{34}\right) e^{\left(-\frac{\lambda}{2}+\frac{\tau}{2}\right)} \widehat{P}_{0}, \\
\widehat{\mathfrak{S}}_{3} & =i\left(-\phi_{13}-\phi_{34}\right) \frac{\delta}{\delta \tau}+\frac{i}{2}\left(\phi_{22}+\phi_{34}+\phi_{13}\right) \frac{\delta}{\delta \lambda}+2 i\left(3 \phi_{22}-2 \phi_{34}\right) \frac{\delta}{\delta P_{0}} \\
& +\left(-\phi_{23}-\phi_{31}\right) e^{\left(-\frac{\lambda}{2}+\frac{\tau}{2}\right)} \widehat{P}_{0}, \\
\widehat{\mathfrak{S}}_{4} & =i\left(\phi_{14}-\phi_{33}\right) \frac{\delta}{\delta \tau}+\frac{i}{2}\left(-\phi_{21}+\phi_{33}-\phi_{14}\right) \frac{\delta}{\delta \lambda}+2 i\left(-3 \phi_{21}-2 \phi_{33}\right) \frac{\delta}{\delta P_{0}} \\
& +\left(\phi_{24}-\phi_{32}\right) e^{\left(-\frac{\lambda}{2}+\frac{\tau}{2}\right)} \widehat{P}_{0},
\end{aligned}
$$

the components (72) $-(75)$ of the supersymmetric constraint $\widehat{\mathfrak{S}}$ can be written in compact form as

$$
\widehat{\mathfrak{S}}_{\mathcal{A}}=i M_{\mathcal{A} 1} \frac{\delta}{\delta \tau}+i M_{\mathcal{A} 2} \frac{\delta}{\delta \lambda}+i M_{\mathcal{A} 3} \frac{\delta}{\delta P_{0}}+M_{\mathcal{A} 4} e^{\left(-\frac{\lambda}{2}+\frac{\tau}{2}\right)} \widehat{P}_{0},
$$

or equivalently

$$
\widehat{\mathfrak{S}}_{\mathcal{A}}=i \Gamma^{1} \frac{\delta}{\delta \tau}+i \Gamma^{2} \frac{\delta}{\delta \lambda}+i \Gamma^{3} \frac{\delta}{\delta P_{0}}+\Gamma^{4} e^{\left(-\frac{\lambda}{2}+\frac{\tau}{2}\right)} \widehat{P}_{0} .
$$

As can be easily seen, we need to find a matrix realization consisting of a set of four independent matrices satisfying the algebra $\left\{\Gamma^{A}, \Gamma^{B}\right\}=0$, for $A \neq B=1, \cdots, 4$. In order to solve the equations $\widehat{\mathfrak{S}}_{\mathcal{A}} \Psi_{\mathcal{A}}=0$ for the polarized case, we use the following $4 \times 4$ matrix realization of the $\Gamma^{A}$ matrices:

$$
\Gamma^{1}=\left(\begin{array}{cccc}
0 & 0 & 0 & i \\
0 & 0 & i & 0 \\
0 & i & 0 & 0 \\
i & 0 & 0 & 0
\end{array}\right), \quad \Gamma^{2}=\left(\begin{array}{cccc}
0 & 0 & 0 & -i \\
0 & 0 & i & 0 \\
0 & -i & 0 & 0 \\
i & 0 & 0 & 0
\end{array}\right),
$$




$$
\Gamma^{3}=\left(\begin{array}{cccc}
0 & 0 & 1 & 0 \\
0 & 0 & 0 & -1 \\
1 & 0 & 0 & 0 \\
0 & -1 & 0 & 0
\end{array}\right), \quad \Gamma^{4}=\left(\begin{array}{cccc}
0 & 0 & -i & 0 \\
0 & 0 & 0 & -i \\
i & 0 & 0 & 0 \\
0 & i & 0 & 0
\end{array}\right),
$$

This choice implies that each $\Psi_{\mathcal{A}}$ splits itself into a four components object. Therefore, the supersymmetric condition reduces to the following set of equations

$$
\begin{array}{r}
i\left[\frac{\delta}{\delta \widehat{P}_{0}}-e^{\left(-\frac{\lambda}{2}+\frac{\tau}{2}\right)} \widehat{P}_{0}\right] \Psi_{\mathcal{A} 3}-\left[\frac{\delta}{\delta \tau}-\frac{\delta}{\delta \lambda}\right] \Psi_{\mathcal{A} 4}=0, \\
-\left[\frac{\delta}{\delta \tau}+\frac{\delta}{\delta \lambda}\right] \Psi_{\mathcal{A} 3}-i\left[\frac{\delta}{\delta \widehat{P}_{0}}+e^{\left(-\frac{\lambda}{2}+\frac{\tau}{2}\right)} \widehat{P}_{0}\right] \Psi_{\mathcal{A} 4}=0, \\
i\left[\frac{\delta}{\delta \widehat{P}_{0}}+e^{\left(-\frac{\lambda}{2}+\frac{\tau}{2}\right)} \widehat{P}_{0}\right] \Psi_{\mathcal{A} 1}-\left[\frac{\delta}{\delta \tau}-\frac{\delta}{\delta \lambda}\right] \Psi_{\mathcal{A} 2}=0, \\
-\left[\frac{\delta}{\delta \tau}+\frac{\delta}{\delta \lambda}\right] \Psi_{\mathcal{A} 1}-i\left[\frac{\delta}{\delta \widehat{P}_{0}}-e^{\left(-\frac{\lambda}{2}+\frac{\tau}{2}\right)} \widehat{P}_{0}\right] \Psi_{\mathcal{A} 2}=0,
\end{array}
$$

or equivalently

$$
\begin{array}{ll}
{\left[\frac{\delta}{\delta \tau}+\frac{\delta}{\delta \lambda}\right] \Psi_{\mathcal{A} 1}=0,} & {\left[\frac{\delta}{\delta \widehat{P}_{0}}+e^{\left(-\frac{\lambda}{2}+\frac{\tau}{2}\right)} \widehat{P}_{0}\right] \Psi_{\mathcal{A} 1}=0,} \\
{\left[\frac{\delta}{\delta \tau}-\frac{\delta}{\delta \lambda}\right] \Psi_{\mathcal{A} 2}=0,} & {\left[\frac{\delta}{\delta \widehat{P}_{0}}-e^{\left(-\frac{\lambda}{2}+\frac{\tau}{2}\right)} \widehat{P}_{0}\right] \Psi_{\mathcal{A} 2}=0,} \\
{\left[\frac{\delta}{\delta \tau}+\frac{\delta}{\delta \lambda}\right] \Psi_{\mathcal{A} 3}=0,} & {\left[\frac{\delta}{\delta \widehat{P}_{0}}-e^{\left(-\frac{\lambda}{2}+\frac{\tau}{2}\right)} \widehat{P}_{0}\right] \Psi_{\mathcal{A} 3}=0,} \\
{\left[\frac{\delta}{\delta \tau}-\frac{\delta}{\delta \lambda}\right] \Psi_{\mathcal{A} 4}=0,} & {\left[\frac{\delta}{\delta \widehat{P}_{0}}+e^{\left(-\frac{\lambda}{2}+\frac{\tau}{2}\right)} \widehat{P}_{0}\right] \Psi_{\mathcal{A} 4}=0 .}
\end{array}
$$

It is straightforward to see that only Eqs. (84) and (86) can be consistently solved. Therefore, the physical state reads

$$
\begin{aligned}
& \Psi_{\mathcal{A} 1}=\Psi_{\mathcal{A} 10} \exp [m(\lambda-\tau)] \exp \left[-e^{\left(-\frac{\lambda}{2}+\frac{\tau}{2}\right)} \frac{\widehat{P}_{0}^{2}}{2}\right] \\
& \Psi_{\mathcal{A} 2}=0 \\
& \Psi_{\mathcal{A} 3}=\Psi_{\mathcal{A} 30} \exp [m(\lambda-\tau)] \exp \left[e^{\left(-\frac{\lambda}{2}+\frac{\tau}{2}\right)} \frac{\widehat{P}_{0}^{2}}{2}\right] \\
& \Psi_{\mathcal{A} 4}=0
\end{aligned}
$$

or equivalently

$$
\Psi_{\mathcal{A}}=\exp [m(\lambda-\tau)]\left(\begin{array}{c}
\Psi_{\mathcal{A} 10} \exp \left[-e^{\left(-\frac{\lambda}{2}+\frac{\tau}{2}\right)} \frac{\widehat{P}_{0}^{2}}{2}\right] \\
0 \\
\Psi_{\mathcal{A} 30} \exp \left[e^{\left(-\frac{\lambda}{2}+\frac{\tau}{2}\right)} \frac{\widehat{P}_{0}^{2}}{2}\right] \\
0
\end{array}\right)
$$


where $\Psi_{\mathcal{A} 10}$ and $\Psi_{\mathcal{A} 30}$ are integration constants and $m$ is a separation constant.

\section{B. The unpolarized case $Q \neq 0$}

Let us now consider the general case of the supersymmetric constraint for the Gowdy $T^{3}$ cosmological models

$$
\begin{aligned}
\widehat{\mathfrak{S}} & =\frac{i}{4}\left\{\left(\gamma^{1} \phi_{1}-\gamma^{3} \phi_{3}\right) \frac{\delta}{\delta \tau}-\frac{i}{2}\left(\gamma^{2} \phi_{2}+\gamma^{3} \phi_{3}-\gamma^{1} \phi_{1}\right) \frac{\delta}{\delta \lambda}-2 i\left(3 \gamma^{2} \phi_{2}-2 \gamma^{3} \phi_{3}\right) \frac{\delta}{\delta P_{0}}\right. \\
& -\gamma^{0} \gamma^{1}\left(\gamma^{3} \phi_{3}-\gamma^{2} \phi_{2}\right) e^{\left(-\frac{\lambda}{2}+\frac{\tau}{2}\right)} \widehat{P}_{0}-4 i\left(\gamma^{2} \phi_{3}-\gamma^{1} \gamma^{2} \gamma^{3} \phi_{1}\right) e^{\widehat{P}_{0}} \frac{\delta}{\delta Q_{0}} \\
& \left.-2 \gamma^{0} \gamma^{2}\left(\gamma^{3} \phi_{1}-\gamma^{1} \phi_{3}\right) e^{\left(-\frac{\lambda}{2}+\frac{\tau}{2}\right)} e^{\widehat{P}_{0}} \widehat{Q}_{0}\right\}
\end{aligned}
$$

As before, the operator $\widehat{\mathfrak{S}}$ has four spinor components:

$$
\begin{aligned}
\widehat{\mathfrak{S}}_{1} & =i\left(-\phi_{11}-\phi_{32}\right) \frac{\delta}{\delta \tau}+\frac{i}{2}\left(-\phi_{24}+\phi_{32}+\phi_{11}\right) \frac{\delta}{\delta \lambda}+2 i\left(-3 \phi_{24}-2 \phi_{32}\right) \frac{\delta}{\delta P_{0}} \\
& +\left(\phi_{21}+\phi_{33}\right) e^{\left(-\frac{\lambda}{2}+\frac{\tau}{2}\right)} \widehat{P}_{0}+4 i\left(-\phi_{13}+\phi_{34}\right) e^{\widehat{P}_{0}} \frac{\delta}{\delta Q_{0}} \\
& +2\left(-\phi_{31}-\phi_{12}\right) e^{\left(-\frac{\lambda}{2}+\frac{\tau}{2}\right)} e^{\widehat{P}_{0}} \widehat{Q}_{0}, \\
\widehat{\mathfrak{S}}_{2} & =i\left(\phi_{12}-\phi_{31}\right) \frac{\delta}{\delta \tau}+\frac{i}{2}\left(\phi_{23}+\phi_{31}-\phi_{12}\right) \frac{\delta}{\delta \lambda}+2 i\left(3 \phi_{23}-2 \phi_{31}\right) \frac{\delta}{\delta P_{0}} \\
& +\left(-\phi_{22}+\phi_{34}\right) e^{\left(-\frac{\lambda}{2}+\frac{\tau}{2}\right)} \widehat{P}_{0}+4 i\left(-\phi_{14}-\phi_{33}\right) e^{\widehat{P}_{0}} \frac{\delta}{\delta Q_{0}} \\
& +2\left(\phi_{32}-\phi_{11}\right) e^{\left(-\frac{\lambda}{2}+\frac{\tau}{2}\right)} e^{\widehat{P}_{0}} \widehat{Q}_{0}, \\
\widehat{\mathfrak{S}}_{3} & =i\left(-\phi_{13}-\phi_{34}\right) \frac{\delta}{\delta \tau}+\frac{i}{2}\left(\phi_{22}+\phi_{34}+\phi_{13}\right) \frac{\delta}{\delta \lambda}+2 i\left(3 \phi_{22}-2 \phi_{34}\right) \frac{\delta}{\delta P_{0}} \\
& +\left(-\phi_{23}-\phi_{31}\right) e^{\left(-\frac{\lambda}{2}+\frac{\tau}{2}\right)} \widehat{P}_{0}+4 i\left(\phi_{11}-\phi_{32}\right) e^{\widehat{P}_{0}} \frac{\delta}{\delta Q_{0}} \\
& +2\left(\phi_{33}+\phi_{14}\right) e^{\left(-\frac{\lambda}{2}+\frac{\tau}{2}\right)} e^{\widehat{P}_{0}} \widehat{Q}_{0}, \\
\widehat{\mathfrak{S}}_{4} & =i\left(\phi_{14}-\phi_{33}\right) \frac{\delta}{\delta \tau}+\frac{i}{2}\left(-\phi_{21}+\phi_{33}-\phi_{14}\right) \frac{\delta}{\delta \lambda}+2 i\left(-3 \phi_{21}-2 \phi_{33}\right) \frac{\delta}{\delta P_{0}} \\
& +\left(\phi_{24}-\phi_{32}\right) e^{\left(-\frac{\lambda}{2}+\frac{\tau}{2}\right)} \widehat{P}_{0}+4 i\left(\phi_{12}+\phi_{31}\right) e^{\widehat{P}_{0}} \frac{\delta}{\delta Q_{0}} \\
& +2\left(-\phi_{34}+\phi_{13}\right) e^{\left(-\frac{\lambda}{2}+\frac{\tau}{2}\right)} e^{\widehat{P}_{0}} \widehat{Q}_{0},
\end{aligned}
$$

once again, the components (94)-(97) of the supersymmetric constraint $\widehat{\mathfrak{S}}$ can be written in compact form as

$$
\begin{aligned}
\widehat{\mathfrak{S}}_{\mathcal{A}} & =M_{\mathcal{A} 1} \frac{\delta}{\delta \tau}+i M_{\mathcal{A} 2} \frac{\delta}{\delta \lambda}+i M_{\mathcal{A} 3} \frac{\delta}{\delta P_{0}}+M_{\mathcal{A} 4} e^{\left(-\frac{\lambda}{2}+\frac{\tau}{2}\right)} \widehat{P}_{0}+i M_{\mathcal{A} 5} e^{\widehat{P}_{0}} \frac{\delta}{\delta Q_{0}} \\
& +M_{\mathcal{A} 6} e^{\left(-\frac{\lambda}{2}+\frac{\tau}{2}\right)} e^{\widehat{P}_{0}} \widehat{Q}_{0},
\end{aligned}
$$


or equivalently

$$
\begin{aligned}
\widehat{\mathfrak{S}}_{\mathcal{A}} & =i \Gamma^{1} \frac{\delta}{\delta \tau}+i \Gamma^{2} \frac{\delta}{\delta \lambda}+i \Gamma^{3} \frac{\delta}{\delta P_{0}}+\Gamma^{4} e^{\left(-\frac{\lambda}{2}+\frac{\tau}{2}\right)} \widehat{P}_{0}+i \Gamma^{5} e^{\widehat{P}_{0}} \frac{\delta}{\delta Q_{0}} \\
& +\Gamma^{6} e^{\left(-\frac{\lambda}{2}+\frac{\tau}{2}\right)} e^{\widehat{P}_{0}} \widehat{Q}_{0}
\end{aligned}
$$

It is straightforward to see that we need to find a matrix realization consisting of a set of six independent matrices satisfying the algebra $\left\{\Gamma^{A}, \Gamma^{B}\right\}=0$, for $A \neq B=1, \cdots, 6$. In order to solve the equations $\widehat{\mathfrak{S}}_{\mathcal{A}} \Psi_{\mathcal{A}}=0$ for the unpolarized case. We use the following $8 \times 8$ matrix realization of the $\Gamma^{A}$ matrices:

$$
\Gamma^{1}=\left(\begin{array}{cccccccc}
0 & 0 & 0 & 0 & 0 & 0 & 0 & -1 \\
0 & 0 & 0 & 0 & 0 & 0 & 1 & 0 \\
0 & 0 & 0 & 0 & 0 & -1 & 0 & 0 \\
0 & 0 & 0 & 0 & 1 & 0 & 0 & 0 \\
0 & 0 & 0 & -1 & 0 & 0 & 0 & 0 \\
0 & 0 & 1 & 0 & 0 & 0 & 0 & 0 \\
0 & -1 & 0 & 0 & 0 & 0 & 0 & 0 \\
1 & 0 & 0 & 0 & 0 & 0 & 0 & 0
\end{array}\right), \quad \Gamma^{2}=\left(\begin{array}{ccccccccc}
0 & 0 & 0 & 0 & 0 & 0 & 0 & 1 \\
0 & 0 & 0 & 0 & 0 & 0 & -1 & 0 \\
0 & 0 & 0 & 0 & 0 & -1 & 0 & 0 \\
0 & 0 & 0 & 0 & 1 & 0 & 0 & 0 \\
0 & 0 & 0 & 1 & 0 & 0 & 0 & 0 \\
0 & 0 & -1 & 0 & 0 & 0 & 0 & 0 \\
0 & -1 & 0 & 0 & 0 & 0 & 0 & 0 \\
1 & 0 & 0 & 0 & 0 & 0 & 0 & 0
\end{array}\right),
$$




$$
\Gamma^{5}=\left(\begin{array}{cccccccc}
0 & 0 & 0 & 0 & 0 & -1 & 0 & 0 \\
0 & 0 & 0 & 0 & -1 & 0 & 0 & 0 \\
0 & 0 & 0 & 0 & 0 & 0 & 0 & -1 \\
0 & 0 & 0 & 0 & 0 & 0 & -1 & 0 \\
0 & -1 & 0 & 0 & 0 & 0 & 0 & 0 \\
-1 & 0 & 0 & 0 & 0 & 0 & 0 & 0 \\
0 & 0 & 0 & -1 & 0 & 0 & 0 & 0 \\
0 & 0 & -1 & 0 & 0 & 0 & 0 & 0
\end{array}\right), \quad \Gamma^{6}=\left(\begin{array}{cccccccc}
0 & 0 & 0 & 0 & 0 & -i & 0 & 0 \\
0 & 0 & 0 & 0 & i & 0 & 0 & 0 \\
0 & 0 & 0 & 0 & 0 & 0 & 0 & i \\
0 & 0 & 0 & 0 & 0 & 0 & -i & 0 \\
0 & -i & 0 & 0 & 0 & 0 & 0 & 0 \\
i & 0 & 0 & 0 & 0 & 0 & 0 & 0 \\
0 & 0 & 0 & i & 0 & 0 & 0 & 0 \\
0 & 0 & -i & 0 & 0 & 0 & 0 & 0
\end{array}\right)
$$

This choice implies that each $\Psi_{\mathcal{A}}$ splits itself into an eight components object. Therefore, the supersymmetric condition $\widehat{\mathfrak{S}}_{\mathcal{A}} \Psi_{\mathcal{A}}=0$ reduces to the following set of equations

$$
\begin{aligned}
& {\left[\frac{\delta}{\delta \tau}+\frac{\delta}{\delta \lambda}\right] \Psi_{\mathcal{A} 1}=0,\left[\frac{\delta}{\delta \widehat{P}_{0}}+e^{\left(-\frac{\lambda}{2}+\frac{\tau}{2}\right)} \widehat{P}_{0}\right] \Psi_{\mathcal{A} 1}=0,\left[\frac{\delta}{\delta Q_{0}}-e^{\left(-\frac{\lambda}{2}+\frac{\tau}{2}\right)} \widehat{Q}_{0}\right] \Psi_{\mathcal{A} 1}=0(103)} \\
& {\left[\frac{\delta}{\delta \tau}+\frac{\delta}{\delta \lambda}\right] \Psi_{\mathcal{A} 2}=0,\left[\frac{\delta}{\delta \widehat{P}_{0}}-e^{\left(-\frac{\lambda}{2}+\frac{\tau}{2}\right)} \widehat{P}_{0}\right] \Psi_{\mathcal{A} 2}=0,\left[\frac{\delta}{\delta Q_{0}}+e^{\left(-\frac{\lambda}{2}+\frac{\tau}{2}\right)} \widehat{Q}_{0}\right] \Psi_{\mathcal{A} 2}=0(104)} \\
& {\left[\frac{\delta}{\delta \tau}-\frac{\delta}{\delta \lambda}\right] \Psi_{\mathcal{A} 3}=0,\left[\frac{\delta}{\delta \widehat{P}_{0}}+e^{\left(-\frac{\lambda}{2}+\frac{\tau}{2}\right)} \widehat{P}_{0}\right] \Psi_{\mathcal{A} 3}=0,\left[\frac{\delta}{\delta Q_{0}}+e^{\left(-\frac{\lambda}{2}+\frac{\tau}{2}\right)} \widehat{Q}_{0}\right] \Psi_{\mathcal{A} 3}=0(105)} \\
& {\left[\frac{\delta}{\delta \tau}-\frac{\delta}{\delta \lambda}\right] \Psi_{\mathcal{A} 4}=0,\left[\frac{\delta}{\delta \widehat{P}_{0}}-e^{\left(-\frac{\lambda}{2}+\frac{\tau}{2}\right)} \widehat{P}_{0}\right] \Psi_{\mathcal{A} 4}=0,\left[\frac{\delta}{\delta Q_{0}}-e^{\left(-\frac{\lambda}{2}+\frac{\tau}{2}\right)} \widehat{Q}_{0}\right] \Psi_{\mathcal{A} 4}=0(106)} \\
& {\left[\frac{\delta}{\delta \tau}+\frac{\delta}{\delta \lambda}\right] \Psi_{\mathcal{A} 5}=0,\left[\frac{\delta}{\delta \widehat{P}_{0}}-e^{\left(-\frac{\lambda}{2}+\frac{\tau}{2}\right)} \widehat{P}_{0}\right] \Psi_{\mathcal{A} 5}=0,\left[\frac{\delta}{\delta Q_{0}}-e^{\left(-\frac{\lambda}{2}+\frac{\tau}{2}\right)} \widehat{Q}_{0}\right] \Psi_{\mathcal{A} 5}=0(107)} \\
& {\left[\frac{\delta}{\delta \tau}+\frac{\delta}{\delta \lambda}\right] \Psi_{\mathcal{A} 6}=0,\left[\frac{\delta}{\delta \widehat{P}_{0}}+e^{\left(-\frac{\lambda}{2}+\frac{\tau}{2}\right)} \widehat{P}_{0}\right] \Psi_{\mathcal{A} 6}=0,\left[\frac{\delta}{\delta Q_{0}}+e^{\left(-\frac{\lambda}{2}+\frac{\tau}{2}\right)} \widehat{Q}_{0}\right] \Psi_{\mathcal{A} 6}=0(108)} \\
& {\left[\frac{\delta}{\delta \tau}-\frac{\delta}{\delta \lambda}\right] \Psi_{\mathcal{A} 7}=0,\left[\frac{\delta}{\delta \widehat{P}_{0}}-e^{\left(-\frac{\lambda}{2}+\frac{\tau}{2}\right)} \widehat{P}_{0}\right] \Psi_{\mathcal{A} 7}=0,\left[\frac{\delta}{\delta Q_{0}}+e^{\left(-\frac{\lambda}{2}+\frac{\tau}{2}\right)} \widehat{Q}_{0}\right] \Psi_{\mathcal{A} 7}=0(109)} \\
& {\left[\frac{\delta}{\delta \tau}-\frac{\delta}{\delta \lambda}\right] \Psi_{\mathcal{A} 8}=0,\left[\frac{\delta}{\delta \widehat{P}_{0}}+e^{\left(-\frac{\lambda}{2}+\frac{\tau}{2}\right)} \widehat{P}_{0}\right] \Psi_{\mathcal{A} 8}=0,\left[\frac{\delta}{\delta Q_{0}}-e^{\left(-\frac{\lambda}{2}+\frac{\tau}{2}\right)} \widehat{Q}_{0}\right] \Psi_{\mathcal{A} 8}=0(110)}
\end{aligned}
$$

It is straightforward to see that only Eqs. (103), (104), (107), and (108) can be consistently 
solved. Therefore, the physical state reads

$$
\begin{aligned}
& \Psi_{\mathcal{A} 1}=\Psi_{\mathcal{A} 10} \exp [m(\lambda-\tau)] \exp \left[-e^{\left(-\frac{\lambda}{2}+\frac{\tau}{2}\right)}\left(\frac{\widehat{P}_{0}^{2}-\widehat{Q}_{0}^{2}}{2}\right)\right], \\
& \Psi_{\mathcal{A} 2}=\Psi_{\mathcal{A} 20} \exp [m(\lambda-\tau)] \exp \left[-e^{\left(-\frac{\lambda}{2}+\frac{\tau}{2}\right)}\left(\frac{-\widehat{P}_{0}^{2}+\widehat{Q}_{0}^{2}}{2}\right)\right], \\
& \Psi_{\mathcal{A} 3}=0 \\
& \Psi_{\mathcal{A} 4}=0 \\
& \Psi_{\mathcal{A} 5}=\Psi_{\mathcal{A} 50} \exp [m(\lambda-\tau)] \exp \left[e^{\left(-\frac{\lambda}{2}+\frac{\tau}{2}\right)}\left(\frac{\widehat{P}_{0}^{2}+\widehat{Q}_{0}^{2}}{2}\right)\right] \\
& \Psi_{\mathcal{A} 6}=\Psi_{\mathcal{A} 60} \exp [m(\lambda-\tau)] \exp \left[-e^{\left(-\frac{\lambda}{2}+\frac{\tau}{2}\right)}\left(\frac{\widehat{P}_{0}^{2}+\widehat{Q}_{0}^{2}}{2}\right)\right], \\
& \Psi_{\mathcal{A} 7}=0 \\
& \Psi_{\mathcal{A} 8}=0
\end{aligned}
$$

or equivalently

$$
\Psi_{\mathcal{A}}=\exp [m(\lambda-\tau)]\left(\begin{array}{c}
\Psi_{\mathcal{A} 10} \exp \left[-e^{\left(-\frac{\lambda}{2}+\frac{\tau}{2}\right)}\left(\frac{\widehat{P}_{0}^{2}-\widehat{Q}_{0}^{2}}{2}\right)\right] \\
\Psi_{\mathcal{A} 20} \exp \left[-e^{\left(-\frac{\lambda}{2}+\frac{\tau}{2}\right)}\left(\frac{\widehat{P}_{0}^{2}+\widehat{Q}_{0}^{2}}{2}\right)\right] \\
0 \\
0 \\
\Psi_{\mathcal{A} 50} \exp \left[e^{\left(-\frac{\lambda}{2}+\frac{\tau}{2}\right)}\left(\frac{\widehat{P}_{0}^{2}+\widehat{Q}_{0}^{2}}{2}\right)\right] \\
\Psi_{\mathcal{A} 60} \exp \left[-e^{\left(-\frac{\lambda}{2}+\frac{\tau}{2}\right)}\left(\frac{\widehat{P}_{0}^{2}+\widehat{Q}_{0}^{2}}{2}\right)\right] \\
0 \\
0
\end{array}\right),
$$

where $\Psi_{\mathcal{A} 10}, \Psi_{\mathcal{A} 20}, \Psi_{\mathcal{A} 50}$ and $\Psi_{\mathcal{A} 60}$ are integration constants and $m$ is, as before, a separation constant. Fig. 1 shows explicitly the behavior of the solution (119).

\section{FINAL REMARKS AND CONCLUSIONS}

The common concern about the use of this formalism is whether the final result of the quantization (in our case, the state function of the universe) depends on the choice of a particular foliation. To clarify this issue in the present case let us consider another choice of the spacetime foliation, i.e., a new time coordinate $t$ defined by

$$
d t=e^{-\frac{1}{4}(\lambda+3 \tau)} d \tau .
$$



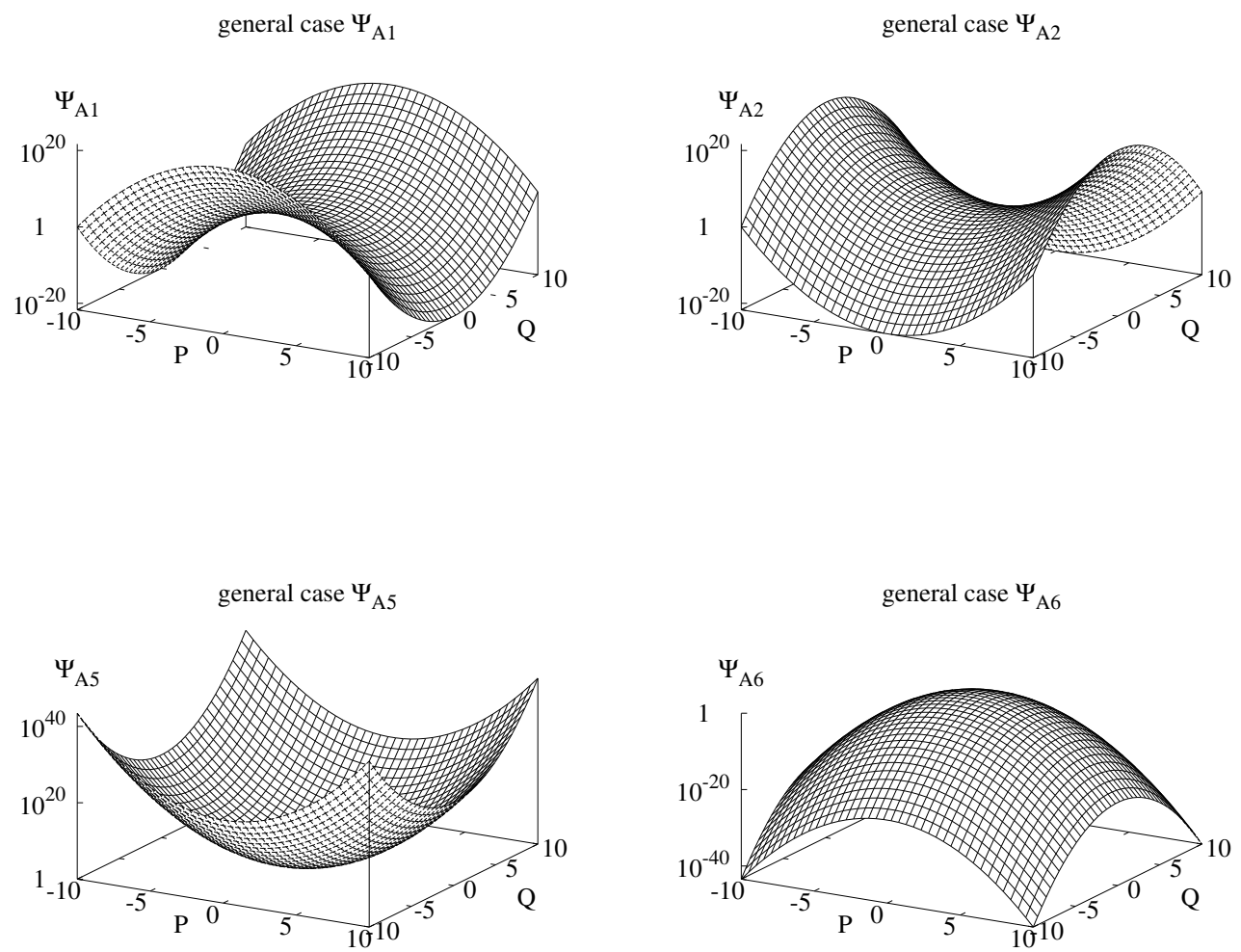

FIG. 1: Behavior of the non-vanishing components of the state function of the universe $\Psi_{\mathcal{A} i}$ with respect to $P_{0}$ and $Q_{0}$ for the special solution (119). We fixed $\tau$ and $\lambda$ to a constant value.

Then, from the general line element (44) we obtain

$$
d s^{2}=d t^{2}-e^{2 \Lambda} d \chi^{2}-e^{F}\left(e^{P} d \sigma^{2}+e^{-P} d \delta^{2}\right),
$$

where $\Lambda=\Lambda(t, \chi)$ and $F=F(t, \chi)$. For the sake of simplicity we are considering here the polarized case only $(Q=0)$. In this particular parametrization the lapse function becomes a constant. Consequently, the problem of the frozen time of the canonical quantization cannot be solved in the framework of our present analysis.

It is dangerous to draw conclusions from some models, minisuperspace or even midisuperspace ones, to full quantum gravity. One should try to avoid common practice, which consists of solving a time problem for a model way down in the hierarchy, and jumping to the conclusion that the time problems of quantum gravity are removed by the same treatment.

On the other hand, it is important to emphasize that the physical interpretation of the 
wave function of the universe $|\Psi\rangle$ presents certain difficulties. A genuine wave function must be related to observable quantities and this implies that $|\Psi\rangle$ must yield a probability density. However, this is not true in this case, since the wave function of the universe is not normalizable. Moreover, if we require that $|\Psi\rangle$ yields a probability density for the 3geometry, which, as it is usual in quantum field theory, must have a specific value at a given time, this would imply a violation of the Hamiltonian constraint [13]. These difficulties in the interpretation of the state function of the universe are the price one has to pay for the use of the canonical quantization procedure, and its inherent a preferred foliation, i.e., the isolation of a specific absolute time parameter against which the evolution of the system should be defined. An alternative procedure like the Dirac quantization, based on functional integrals, does not require to single out the time variable and could lead to a quantum system with less interpretation difficulties [40]. Nevertheless, even this other Dirac approach does not solve the time arbitrariness problem.

In this work we have investigated the quantization of Gowdy $T^{3}$ cosmological models in the context of $N=1$ supergravity. The quantum constraints, resulting from the canonical quantization formalism, are explicitly analyzed and solved. In this way, we find the state function of the universe for the polarized and unpolarized Gowdy $T^{3}$ models. This represents a proof of the existence of physical states in the $(N=1)$ supersymmetric simple midisuperspace, corresponding to Gowdy cosmologies. This result contrasts drastically with analogous investigations in minisuperspace (Bianchi-like) models, where no physical states exist, a result that sometimes is assumed as a sufficient proof to dismiss $N=1$ supergravity. We have adopted a less radical position in this work and dismiss as non-physical only the homogeneous minisuperspace models. The existence of physical states in midisuperspace models confirms this conclusion and indicates that $N=1$ supergravity is a valuable theory which should be investigated further. In this context we have also obtained an interesting result showing that, in the Gowdy $T^{3}$ midisuperspace model analyzed in this work, the state function of the universe, representing nontrivial physical states is completely free of anomalies.

On the other hand, there exists a belief that the second quantization solves the problem of time in quantum theory of a relativistic particle. The second quantization approach to quantum field theory is based on the construction of a Fock space, i.e., one takes a oneparticle Hilbert space $\mathcal{F}_{(1)}$. From the direct product of the one-particle states the states 
which span the $\mathrm{N}$-particle sector $\mathcal{F}_{(N)}$ are constructed. The Fock space $\mathcal{F}$ is then the direct sum of all such sectors, i.e., $\mathcal{F}=\mathcal{F}_{(0)} \oplus \mathcal{F}_{(1)} \oplus \mathcal{F}_{(2)} \oplus \cdots$, where $\mathcal{F}_{(0)}$ is spanned by the vacuum state. It is clear that the Fock space $\mathcal{F}$ can be defined only if the one-particle state $\mathcal{F}_{(1)}$ is a Hilbert space. This brings us to the Hilbert space problem for a relativistic particle. The absence of a privileged one-particle Hilbert space structure is source of ambiguities in constructing a unique quantum field theory on a dynamical background [9].

In full, the second quantization merely shifts the problem of the arbitrariness of time to a different level without really solving it. Consequently, our quantization approach à la Pilati [7] remains valid since the second quantization does not represent a significant improvement to the quantization approach regarding the time evolution problem.

A closer look to the second quantization approach reveals that it does not really solve the problem of time evolution and its formalism resists an operational interpretation, like the problems presented by the indefinite inner product of the Klein-Gordon interpretation, which are faced by suggesting that the solutions of the Wheeler-DeWitt equation are to be turned to operators. This is analogous to subjecting the relativistic particle, whose state is described by the Klein-Gordon equation, to second quantization.

In this work we have focused on the special case of $T^{3}$ cosmologies. The generalization of our results to include the case of $S^{1} \times S^{2}$ Gowdy models seems to be straightforward. In particular, we believe that the unified parametrization introduced in [41], which contains both types of topologies, could be useful to explore the supersymmetric Gowdy model in quite general terms.

\section{Acknowledgments}

This research was supported by CONACyT Grants 48404-F and 47000-F, and by the collaboration Mexico-Germany, grants CONACyT-DFG J110.491 and J110.492.

[1] C. W. Misner, in: Magic without Magic: John Archibald Wheeler, ed. J. R. Klauder (Freeman, San Francisco, 1972).

[2] M.P. Ryan: Hamiltonian Cosmology (Springer, New York, 1972). 
[3] D.Z. Freedman, P. van Nieuwenhuizen, and S. Ferrara, Progress toward a theory of supergravity, Phys. Rev. D13 3214 (1976).

[4] C. Teitelboim, Surface integrals as symmetry generators in supergravity theory, Phys. Lett. B69 240 (1977).

[5] C. Teitelboim, Supergravity and Square Roots of Constraints, Phys. Rev. Lett. 381106 (1977).

[6] R. Tabensky and C. Teitelboim, The square root of general relativity, Phys. Lett. B69 453 (1977).

[7] M. Pilati, The canonical formulation of supergravity, Nucl. Phys. B132 138 (1978).

[8] A. Macías, O. Obregón and M. P. Ryan, Quantum Cosmology: The Supersymmetric Square Root, Class. Quantum Grav. 41477 (1987).

[9] K. Kuchař, Time and interpretation of quantum gravity, in: Proc. 4th Canadian Conf. on General Relativity and Relativistic Astrophysics, eds. G. Kunstatter, D. Vincent, and J. Williams (World Scientific, Singapore, 1992) pp. 211-314.

[10] A. Macías and H. Quevedo, Time paradox in quantum gravity, in: Quantum Gravity - Mathematical models and experimental bounds. B. Fauser, J. Tolksdorf, E. Zeidler eds., (Birkhaeuser, Basel, 2006) pp. 41-60.

[11] B. S. DeWitt, Quantum Theory of Gravity I. The canonical theory. Phys. Rev. 1601113 (1967) 1113; C. W. Misner, Quantum Cosmology. I, Phys. Rev. 1861319 (1969).

[12] M.P. Ryan and L.C. Shepley: Homogeneous Relativistic Cosmologies (Princeton University Press, New Jersey, 1975).

[13] K. V. Kuchař and M. P. Ryan, Is minisuperspace quantization valid? Taub and Mixmaster. Phys. Rev. D40 3982 (1989).

[14] M. Kaku: Quantum Field Theory (Oxford University Press 1993).

[15] P.D. D'Eath, S. Hawking, and O. Obregón, Supersymmetric Bianchi models and the square root of the Wheeler-DeWitt equation. Phys. Lett. B300 44 (1993).

[16] A. Csordás and R. Graham, Supersymmetric Minisuperspace with Nonvanishing Fermion Number, Phys. Rev. Lett. 744129 (1995).

[17] A. Csordás and R. Graham, Hartle-Hawking State in Supersymmetric Minisuperspace, Phys. Lett. B373 51 (1996).

[18] P.D. D'Eath, Canonical quantization of supergravity, Phys. Rev. D29 2199 (1984). Bosonic physical states in $N=1$ supergravity, Phys. Lett. B321 368 (1994). 
[19] O. Obregón, J. Pullin, and M.P. Ryan, Bianchi cosmologies: New variables and a hidden symmetry Phys. Rev. D48 5642 (1993).

[20] A. Macías, O. Obregón and J. Socorro, Supersymmetric Quantum Cosmology. Int. J. Mod. Phys. A8 4291 (1993).

[21] A. Macías, E.W. Mielke, and J. Socorro, Supersymmetric quantum cosmology for Bianchi class A models. Int. J. Mod. Phys. D7 701 (1998).

[22] A. Macías, The different approaches to quantum cosmology. Gen. Rel. Grav. 31653 (1999).

[23] A. Macías, E.W. Mielke, and J. Socorro, Supersymmetric Quantum Cosmology: The Physical States, Phys. Rev. D57 1027 (1998).

[24] D. Kramer, D. Stephani, E. Herlt, M. MacCallum, and E. Schmutzer, Exact Solutions of Einstein's Field Equations. (Cambridge University Press, Cambridge, England, 1980).

[25] A. Ashtekar and M. Pierri, Probing quantum gravity through exactly soluble midisuperspaces I, J. Math. Phys. 376250 (1996).

[26] G. A. Mena-Marugan, Canonical quantization of the Gowdy model, Phys. Rev. D56 908 (1997).

[27] R. Gowdy, Gravitational Waves in Closed Universes, Phys. Rev. Lett. 27826 (1971).

[28] R. Gowdy, Vacuum Space-Times with Two Parameter Spacelike Isometry Groups and Compact Invariant Hypersurfaces: Topologies and Boundary Conditions, Ann. Phys. (N. Y.) 83 203 (1974).

[29] M.F. Sohnius, Introducing supersymmetry, Phys. Rep. 12839 (1985).

[30] J. Wess and B. Zumino, Supergauge transformations in four dimensions Nucl. Phys. B70 39 (1974); A lagrangian model invariant under supergauge transformations, Phys. Lett. B49 52 (1974).

[31] E.W. Mielke, P. Baekler, F.W. Hehl, A. Macías, and H.A. Morales-Técotl, Yang-Mills-Clifford form of the chiral Einstein action, in: Gravity, Particles and Space-Time, ed. by P. Pronin and G. Sardanashvily (World Scientific, Singapore, 1996), pp. 217-254.

[32] E.W. Mielke, E.W, A. Macías, and H.A. Morales-Técotl, Chiral fermions coupled to chiral gravity, Phys. Lett. 215A 14 (1996).

[33] S. Deser, J.H. Kay, and K.S. Stelle, Hamiltonian Formulation of Supergravity, Phys. Rev. D16 2448 (1977).

[34] A. Macías: "Chiral (N=1) supergravity". Class. Quantum Grav. 133163 (1996). 
[35] P. van Nieuwenhuizen, Supergravity, Phys. Rep. 68189 (1981).

[36] C. W. Misner,A minisuperspace Example: The Gowdy $T^{3}$ Cosmology, Phys. Rev. D8 3271 (1973).

[37] B.K. Berger, Quantum Graviton Creation in a Model Universe Ann. of Phys. (NY) 83458 (1974).

[38] A. Sánchez, Ph.D. thesis: Gowdy cosmological models in supergravity. Universidad Autónoma Metropolitana-Iztapalapa, México, 2007 (unpublished); A. Macías, H. Quevedo and A. Sánchez, Local Lorentz invariance in $N=1$ supergravity, Phys. Rev. D73, 027501 (2006).

[39] S.M. Carroll, D. Z. Freedman, M.E. Ortiz, and D. Page, Physical states in canonically quantized supergravity, Nucl. Phys. B423 661 (1994).

[40] J. Guven and M. P. Ryan, Functional integrals and canonical quantum gravity, Phys. Rev. D45 3559 (1992).

[41] H. Quevedo, Acausality in Gowdy spacetimes, Gen. Rel. Grav. 38599 (2006).

[42] These relations are quite general, they do not depend on the particular Bianchi model into consideration, cf. [35] 